\title{
GPS-DENIED GEO-LOCALISATION USING VISUAL ODOMETRY
}

\author{
Ashish Gupta*, Huan Chang, Alper Yilmaz \\ The Ohio State University, Columbus, Ohio, United States - (gupta.637, chang.1522, yilmaz.15)@ osu.edu
}

\author{
Commission III, WG III/3
}

KEY WORDS: GPS-denied, Geo-localisation, 3D view geometry, computer vision, GIS, OpenStreetMap

\begin{abstract}
:
The primary method for geo-localization is based on GPS which has issues of localization accuracy, power consumption, and unavailability. This paper proposes a novel approach to geo-localization in a GPS-denied environment for a mobile platform. Our approach has two principal components: public domain transport network data available in GIS databases or OpenStreetMap; and a trajectory of a mobile platform. This trajectory is estimated using visual odometry and 3D view geometry. The transport map information is abstracted as a graph data structure, where various types of roads are modelled as graph edges and typically intersections are modelled as graph nodes. A search for the trajectory in real time in the graph yields the geo-location of the mobile platform. Our approach uses a simple visual sensor and it has a low memory and computational footprint. In this paper, we demonstrate our method for trajectory estimation and provide examples of geolocalization using public-domain map data. With the rapid proliferation of visual sensors as part of automated driving technology and continuous growth in public domain map data, our approach has the potential to completely augment, or even supplant, GPS based navigation since it functions in all environments.
\end{abstract}

\section{INTRODUCTION}

Autonomous navigation is an emerging technology with a huge potential; self-driving cars are almost round the corner. This technology requires accurate geo-localization in real-time to effectively navigate urban environments. Currently, navigation technologies are GPS reliant, which has multiple issues. The accuracy of standard GPS devices is unacceptably poor for the purposes of autonomous navigation and accurate GPS sensors are very expensive. GPS is unreliable in several environments where transmission between the device and satellites is impeded by surrounding structures, like tall buildings in an urban environment, the so called 'urban canyons'. In addition, GPS is unavailable indoors, underground, in tunnels, etc. and can also be degraded or denied in certain geographic regions. In order to be viable for a huge consumer market, there is a need for geo-localization solution that operates with low-cost sensors and public domain data.

The DARPA grand challenge has demonstrated the effectiveness of visual sensors in autonomous navigation by analyzing visual information for video cameras mounted on the mobile platform. There are arguably two main conceptual approaches for vision based methods. One of these approaches seeks to use an ever growing geo-tagged image database, as in Google Street View. The images from the camera are queried to this database and matches are used to infer the geo-location of the mobile platform in real-time (Vaca-Castano et al., 2012). While such localization results show promise, this approach requires a maintenance and continuous access to very large image databases which is extremely expensive and not currently not a feasible solution (Paul and Newman, 2010). The second approach is inspired by navigation in robotics using SLAM, which perform a localization and mapping of the neighborhood of the robot. The key issue with SLAM framework is the accumulation of drift error that results in poor localization, which is more pronounced for traversals over long distances .

This paper poses the problem of accurate geo-localization as a combination of accurate relative localization within a very large

\footnotetext{
${ }^{*}$ Corresponding author
}

spatial search space. The relative localization is computed using Visual Odometry (VO) and the large spatial search space is acquired from GIS databases. The key insight in this paper is that the motion of the typical mobile platform is correlated to the associated topology of the spatial search space. For example, a car moves on roads and so the trajectory of the car for a finite distance of travel is correlated to a subset of the transport network layer of GIS in the spatial search space for that car. The use of one or more layers of GIS based on the type of mobile platform and sensors facilitates flexibility in our approach and the ability to scale the solution.

The combination of Inertial Measurement Units (IMUs) and visual sensors is a crucial and useful technique for autonomous navigation, particularly in unknown indoor or GPS-denied environments. Although pure monocular vision-based Extended Kalman Filter (EKF) can achieve around meter level of the trajectory error, it is limited in a small or well constraint area in order to avoid scale drift (Civera et al., 2010). Using sequential stereo pairs or camera matrix is capable of handling the scaling problem as a depth measurement based on epipolar geometry, with wellcalibrated camera interior orientation parameters (IOP) and relative exterior orientation parameters (EOP). However, the shutter synchronization, calibration bias and other uncertainties between camera pairs will lead to a camera trajectory drift. A robust onsite calibration with the unknown features in the scenes is still an unsolved problem. In this perspective, using monocular camera visual odometry is more practicable than stereo pairs or other depth measurement device.

Camera and IMU measurement have several complements than other sensor combination, and both of them are commonly mounted in consumer mobile devices nowadays. IMU provides continuously linear accelerations and rotational velocities, which can be integrated as the relative pose displacement. It is reliable in the sort time period, so we can resolve the major drawback of using monocular visual odometry: such as scale estimation, image blur due to rapid motion, and the requirement of continuously feature tracking. On the other hand, IMU-based pose estimation has a critical drawback due to integrating IMUs measurements. The 
noise and bias inherent in these measurements are multiplied and cumulative after each integration, causes the system pose drift to become significantly unbounded over time. To overcome these drawbacks of the IMU drifting problem, the camera is capable of detecting feature points in the scenes and using them as a pose constraint. In synergy, IMU provides short-term reliable system pose for camera to measure unknown features in the scenes as 3D landmarks.

Our contributions in this paper are: (i) we introduce a novel approach to geo-localization of mobile platforms in real-time that combines GIS/OpenStreetMap data and visual sensors on-board the mobile platform. We demonstrate a computationally efficient approach at is scalable to search accurate geo-location in a very large region of interest; and (ii) this study proposed a visualinertial navigation system (VINS), including monocular visual odometry and IMU. Proposed method improved feature tracking and camera measurement results, providing better EKF-based visual-inertial pose estimation.

\section{RELATED WORK}

Global localization is an important part of any autonomous navigation system and its uninterrupted performance is crucial for the stability and robustness of the system. To achieve this goal, several visual odometry based approaches have emerged in recent years, offering high quality localization performance even at real-time execution speeds. The basic pipeline of these methods includes a feature extraction stage, followed by feature matching, 3D reconstruction of a sparse point cloud, and finally estimation of the pose of the mobile platform. A windowed bundle adjustment is typically run at the end. The main drawback of this category of methods is that drift is accumulated over time. As a result, after the mobile platform has travelled a certain distance, the localization error becomes significant, making the localization result unusable over longer distances (Engel et al., 2015). In parallel to the $\mathrm{VO}$ approaches, there are a number of methods that perform visual-SLAM (Lategahn et al., 2011). The vSLAM approach is similar to VO, differing in that reconstructed 3D points are used as landmarks and their position is optimized together with the camera pose.

There has been research towards using map information that is already available in the form of 2D (e.g., Google Maps, OpenStreetMaps) or 3D road maps (e.g., Google Maps 3D) for localization. (Hentschel and Wagner, 2010) propose the use of OpenStreetMap maps to facilitate robot localization and navigation. A cadastral map with the building footprints is extracted and the GPS position of the robot is used in an MCL framework to provide localization for the robot. Recently, (Senlet and Elgammal, 2011) have presented a system that uses Google Static Maps to perform global localization. In particular, they perform a local reconstruction of the scene, using stereo depth maps, which they later compare with the 2D map. Both of the aforementioned approaches show the potential that the use of map information can bring to the localization task. However, these systems require a manual initial localization and have only been tested in suburban areas where the environment is relatively simple and the paths they have travelled are short in length.

Elaborating on VO methods, a majority focus on integrating IMU and monocular vision using filter-based(KF, Information Filter, particle filter), EM-based, or batch optimization (shape from motion) (Strelow and Singh, 2004) methods. Bundle adjustment is a non-linear global optimization approach, which can produces estimates of the sensor motion, 3D points structure of the scenes, and other unknown variables (e.g.. biases) using measurements from the entire observation simultaneously. But the computation cost and complexity is to heavy, especially when the image sequence is long.

EM iterates two major steps, expectation step for posterior system pose from map; and maximization step to find most likely pose from the expectations, and finally build up a series of increasingly accurate maps. EKF-based SLAM is another efficient recursive algorithm. EKF-based approach provides optimal minimum mean-square Error (MMSE) estimates of the state (device and landmark positions), and its covariance matrix seems to converge strongly. However, the Gaussian noise assumption restricts the adaptability of the EM and KF for data association and number of landmarks. Sliding Window Filter (SWF) is a keyframebased operating method, performing a batch optimization over a set number of system states, which is useful for large scale operations. Multi-State Constraint Kalman Filter (MSCKF) (Mourikis and Roumeliotis, 2007) preserve a buffer window of sensor poses and simultaneously update each pose in the window using batchoptimized estimates of observed landmarks across the buffer window. This study implement MSCKF for Camera-IMU trajectory estimation, and improved a more robust landmark feature descriptor for tracking process. Camera-IMU calibration and data synchronization are the priori Information for the developed VINS These calibration parameters are including time scaling and offset, relative pose between IMU and camera, and IMU bias. (Ovrén and Forssén, 2015) proposed a non-linear least squares minimization method using the symmetric transfer error as cost function, joint calibration of a wide-angle rolling shutter camera and an externally mounted gyroscope. Calibration of camera IOP includes principle points, focal length and lens distortion. Observed images then can correct deformation regarding IOP for higher accuracy matching process. Domain-size pooling scale-invariant feature transform (DSP-SIFT) (Dong and Soatto, 2015) improve the robustness of point-based descriptor by pooling gradient orientations across different domain sizes. This kept the dimension remains the same but more appropriate for against photometric nuisances.

\section{METHODOLOGY}

We pose the problem of accurate mobile platform geo-localization as a combination of approximate geo-localization and accurate relative localization. The approach requires an initial hypothesis of region where the mobile platform can be located, which can be arbitrarily large from size of a village to the entire globe, though computational complexity scales with a larger search space. For typical use cases, the region of interest would be a political state or city sized area, which we have selected for our experiments in this paper. Our approach is illustrated in the block diagram in Figure 1.

The first step is to acquire map data from a public domain source like OpenStreetMap (OSM) or Geographic Information System (GIS) databases. OpenStreetMap data can be accessed via the corresponding website, and the user can download the map for the region of interest by specifying a bounding box $\mathbf{b}$ in terms of longitude and latitude, $\mathbf{b}=\left(\right.$ lat $_{\min }$, lon $_{\min }$, lat $_{\max }$, lon $\left._{\max }\right)$. The map is given in XML format and is structured using three basic entities: nodes, ways and relations. The node $\mathbf{n}$ represents a point element in the map and is defined by its GPS coordinates, longitude and latitude $\mathbf{n}=$ (lat, lon). Linear and area elements are represented by ways $\mathbf{w}$, which are formed as a collection of nodes $\mathbf{w}=\left\{n_{i}\right\}_{i=1 \ldots k}$. The relations $\mathbf{r}$ are used to represent relationships between the aforementioned geometric entities to form more complicated structures. The interesting map structures for 


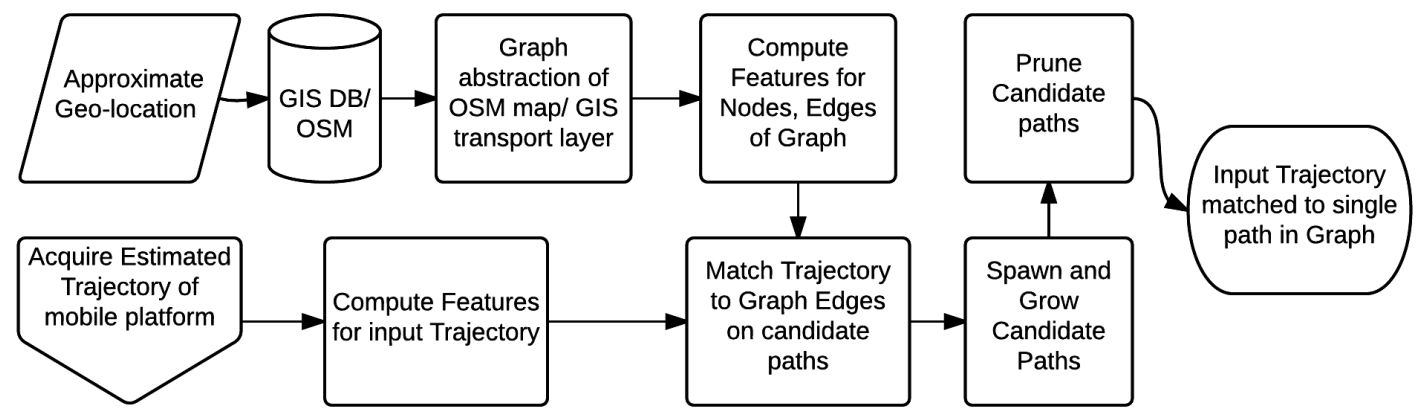

Figure 1: Mobile Trajectory Geo-Localization: VO is used to compute a trajectory in real-time which is abstracted as a sub-graph and is progressively localized by sub-graph matching in a graph abstraction of transport network map of the region of interest.

us are streets, which are modelled as ways, and can be extracted from the XML data in to form a street graph of the region of interest. Map data can also be acquired from GIS databases in the form of 'shapefiles' format, that is a popular geospatial vector data format developed and regulated by Esri. Similar to OSM, the shapefile data is used to construct a road network graph. A example of OpenStreetMap, shapefile and computed transport network graph is shown in Figure 2. Community contributed data sources like OpenStreetMap are being continuously updated whereas shapefile databases are comparatively better curated and updated more infrequently. The choice of data source would depend on the region of interest and the current state of respective maps of that region. In addition, it should be noted that convenience of data access comes with the cost of registration errors and noise which makes the task of searching the mobile platform trajectory in the graph more difficult. The second step is to compute feature descriptors for the 3D trajectory of the mobile platform and the transport network graph. We chose a contour tangent angle $\theta$ as uniform sample distance, illustrated in Figure $4 \mathrm{a}$ for the feature descriptor as a trade-off between demands for computational efficiency in processing and storage, robustness to noise and missing data, and scalability. There exist simple to complex trajectory descriptors with inbuilt invariance and other features, but have a corresponding high computational and storage cost. In order to aid scalability of the solution and simple enough so it can be deployed on mobile computational platforms with comparatively low processing power, we opted for a descriptor that is both fast and good for search trajectory in the graph. A trajectory $T=\left\{P_{1}, P_{2}, \ldots, P_{n}\right\}$ consists of points at roughly uniform sampling distance. The quantization associated with sampling distance is relevant in terms of sensitivity to the motion of the mobile platform (for example, swerve, lane-change and over-taking motion of a car) and also relevant to scalability. Our choice of sampling distance was empirically determined. The motion from point $P_{t}$ to point $P_{t+1}$ is encoded in terms of the angle $\theta$, shown in Figure 3a, and its associated bin. We found quantizing the contour angles to 72 bins, $\theta \in[0, \ldots, 71]$, worked well in our experiments. The choice of quantization level is based on optimizing sensitivity to mobile platform motion while minimizing effect of noise. Figure $4 \mathrm{~b}$ shows a trajectory encoded in this manner.

\subsection{Trajectory Search in Graph}

To find the mobile platform trajectory in the road network, we abstract both to a graph data structure and pose the problem of trajectory search as a sub-graph matching problem, where the graph of the trajectory of a mobile platform travelling on the roads of a map is a sub-graph of the entire map graph. The transport network map $\mathcal{M}$ has been abstracted as a graph $\mathcal{G}_{\mathcal{M}}$, illustrated in Figure 2c. The trajectory $\tau$ is abstracted as a graph $\mathcal{G}_{\tau}$. Graph matching works by computing equivalent features on both map graph $\mathcal{G}_{\mathcal{M}}$ and trajectory graph $\mathcal{G}_{\tau}$ and storing these feature values at the nodes and or edges of the graph, which are then used to compute a similarity score between trajectory graph and a path graph $\mathcal{G}_{\mathcal{P}}$, which is a hypothesis of a matching sub-graph of $\mathcal{G}_{\mathcal{M}}$.

With the aim of facilitating a generalized solution that does not require knowledge of geographic coordinates or global direction information of the mobile platform, we assume that the trajectory estimated by $\mathrm{VO}$ is in its simplistic form a sequence of points with relative distance measure. This means the most reliable mode of encoding the trajectory is change in angle of motion of the mobile platform which is equivalent to contour angles of a spline approximation of the acquired sequence of points. Figure 5 illustrates an acquired trajectory as a set of points $T_{V O}$. We compute a spline approximation using these points and those points with significant change in contour angle are selected as 'control points'. A graph abstraction of this trajectory $\mathcal{G}_{\tau}$ is created using the control points as nodes of the graph and set of points between control points comprise an edge of the graph, wherein are computing $T_{V O} \rightarrow \mathcal{G}_{\tau}$. Since the mobile platform travels on the road network the trajectory graph can be assumed to be a sub-graph of the map graph, $\mathcal{G}_{\tau} \subset \mathcal{G}_{\mathcal{M}}$. Our approach to searching for a match in $\mathcal{G}_{\mathcal{M}}$ is to introduce 'path graph' $\mathcal{G}_{\mathcal{P}}$. Searching for $\mathcal{G}_{\tau} \subset \mathcal{G}_{\mathcal{M}}$ is equivalent to search for a sub-graph $\mathcal{G}_{\mathcal{P}}$ of $\mathcal{G}_{\mathcal{M}}$ such that the trajectory is similar to this path sub-graph $\mathcal{G}_{\tau} \equiv \mathcal{G}_{\mathcal{P}}$. So, we compute a similarity score $\mathcal{S}(\cdot, \cdot)$ between the trajectory graph and path graph, $\mathcal{S}\left(\mathcal{G}_{\tau}, \mathcal{G}_{\mathcal{P}}\right) \forall \mathcal{G}_{\mathcal{P}} \subset \mathcal{G}_{\mathcal{M}}$, for all path graphs in the map graph. The best matching path graph is

$$
\mathcal{G}_{\mathcal{P}}^{*} \leftarrow \underset{\mathcal{G}_{\mathcal{P}}}{\arg \max } \mathcal{S}\left(\mathcal{G}_{\tau}, \mathcal{G}_{\mathcal{P}}\right) \forall \mathcal{G}_{\mathcal{P}} \subset \mathcal{G}_{\mathcal{M}}
$$

We are considering the problem of geo-localization in real-time. The estimated trajectory $T_{V O}$ continuously grows with time and consequently the trajectory graph also grows with time. Consequently we formulate our trajectory search solution accordingly, where best matching solution at time $t$ is given in equation 1 .

$$
\mathcal{G}_{\mathcal{P}}^{(t) *} \leftarrow \underset{\mathcal{G}_{\mathcal{P}}^{(t)}}{\arg \max } \mathcal{S}\left(\mathcal{G}_{\tau}^{(t)}, \mathcal{G}_{\mathcal{P}}^{(t)}\right) \forall \mathcal{G}_{\mathcal{P}}^{(t)} \subset \mathcal{G}_{\mathcal{M}}
$$

In our implementation $\mathcal{S}(\cdot, \cdot)$ is computed using a sub-string matching algorithm. The trajectory $\mathcal{G}_{\tau}^{(t)}$ is a sequence of quantized edge-orientations we described earlier. Each graph path $\mathcal{G}_{\mathcal{P}}$ is also similarly encoded as a sequence of quantized edge-orientations. 


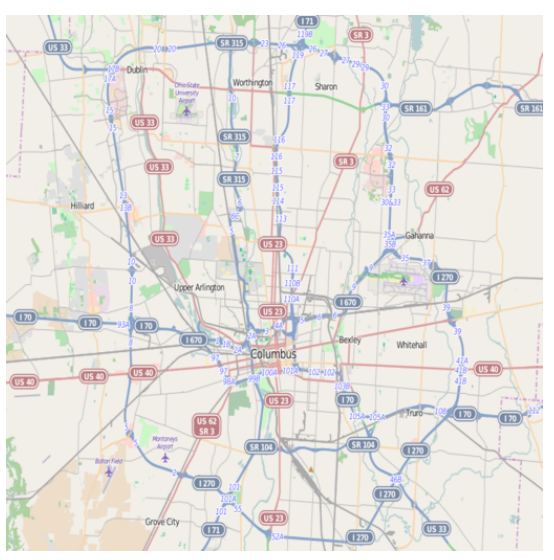

(a) OpenStreetMap

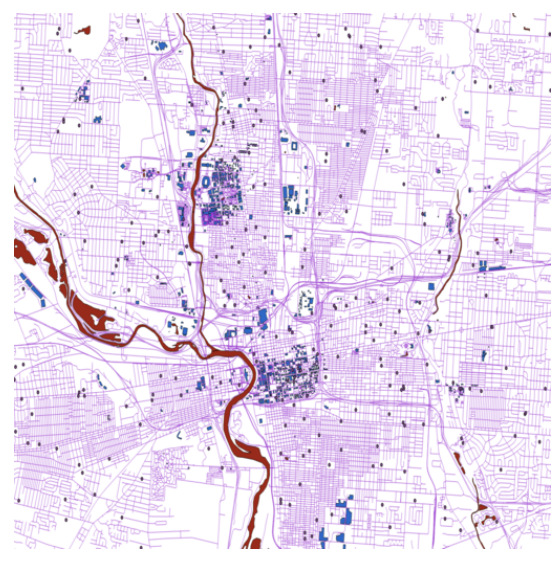

(b) GIS ShapeFile

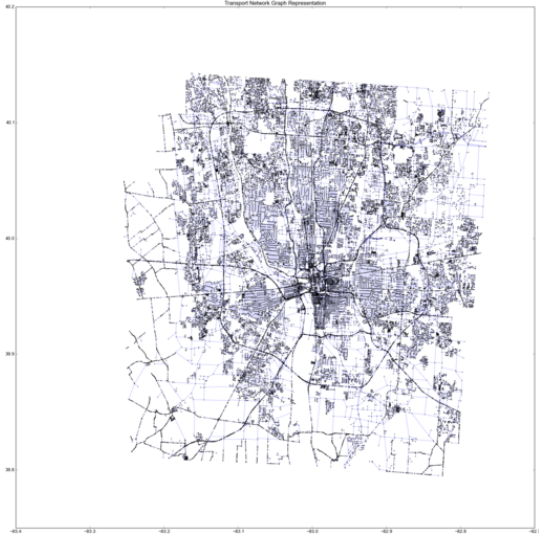

(c) Graph representation

Figure 2: Abstraction of OpenStreetMap or GIS data to a transport network Graph representation

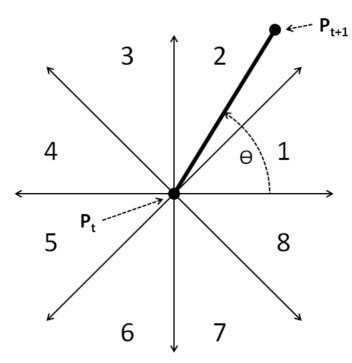

(a) Encoding trajectory of mobile platform and edges on graph of transport net- (b) Features for undirected work. The motion from point graph are computed as angles $P_{t}$ at sampling interval $t$ to between each pair of edges at $P_{t+1}$ is measured in angle $\theta$ each node $N_{0}$ with its neighwhich is quantized to corre- bors $\mathcal{N}=\left\{N_{1}, N_{2}, N_{3}\right\}$. sponding bin. (This illustra- The features $\left[\theta_{10}^{02}, \theta_{20}^{03}, \theta_{30}^{01}\right]$ is tion uses 8 bins for visual- stored at each node of the ization, though in our imple- graph $\mathcal{G}_{\mathcal{M}}$ mentation we use 72 bins.)

Figure 3: Encoding mobile platform trajectory and graph.

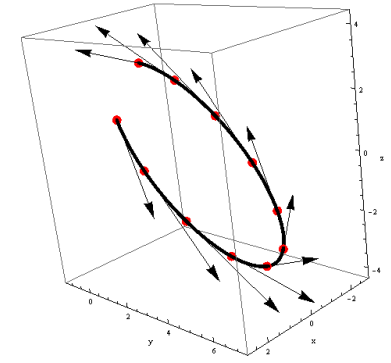

(a) 3D trajectory descriptor

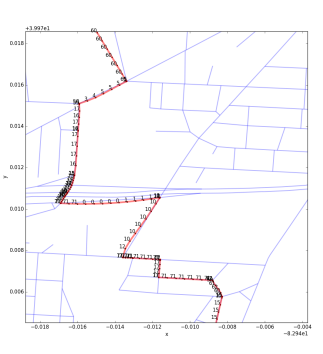

(b) Road graph descriptor
Figure 4: Feature descriptor for estimated 3D trajectory of mobile platform and edges of graph representing roads. The descriptor is computationally efficient and facilitates matching between the trajectory of the mobile platform and the transport network graph.

The key benefit of using string matching is that partial matches also produce a reasonably good similarity score. This formulation makes $\mathcal{S}(\cdot, \cdot)$ robust to noise, missing data, erroneous vehicle motion like swerving, map registration flaws, etc. The motivation for this approach is derived from the popular Bag-of-Words method

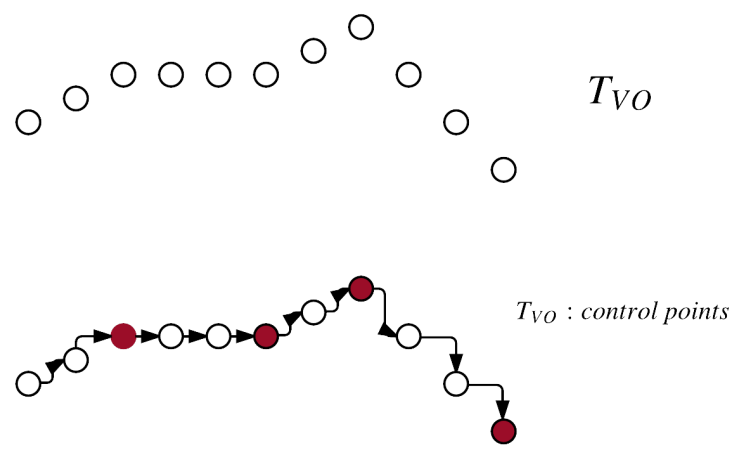

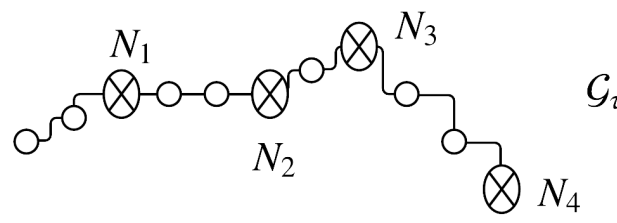

Figure 5: The trajectory estimated from visual odometry $T_{V O}$ is a sequence of points with relative vector information. A subset of these points where there is significant change in contour are selected a 'control points', which become nodes in a trajectory $\operatorname{graph} \mathcal{G}_{\tau}$

in computer vision that has also been used for SLAM (Paul and Newman, 2010).

\subsection{Visual Odometry}

We propose an efficient camera pose trajectory-tracking algorithm combining vision-based motion estimation and IMU data. The developed procedure for VINS is shown in Figure 6. The first step is to decimate the IMU readings in order to synchronize with camera frames with the same measurement frequency and smooth IMU noise. Captured images are then processed with tracking algorithm described in section 3.2.1. The camera initial pose was given by IMU readings, and then, vision-based $3 \mathrm{D}$ point triangulation were used to estimate feature points coordinates. After repeated measurement, reliable feature points can be used to update camera position using EKF as mentioned in section 3.2.2. 


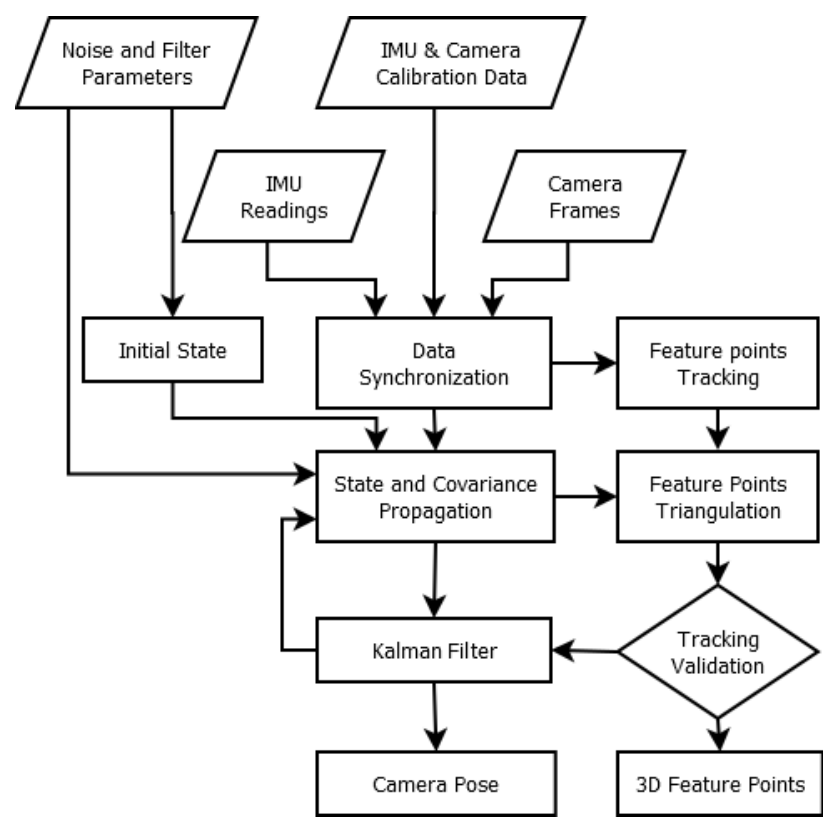

Figure 6: Visual Odometry

3.2.1 Feature detection and tracking Feature extraction and tracking is the essential work for vision-based mapping and trajectory estimation. Scale-invariant feature transform (SIFT) is a well-known feature point descriptor, which was designed to reduce variability due to illumination while retaining discriminative power in the scale space. Each un-normalized cell of SIFT can be written as:

$h_{S}(\theta, I, \sigma)(x)=\int E_{\epsilon}(\angle \nabla I(y)-\theta) E_{\sigma}(y-x)\|\nabla I(y)\| d y$,

where $I$ is the image window of the centred pixel $x$ with spatial pooling scale $\sigma . \theta$ is corresponding to orientation histogram bin $(\epsilon)$, normally with 8 direction from 0 to $2 \pi$. Kernel $E_{\epsilon}$ and $E_{\text {sigma }}$ represents a bilinear of size $\epsilon$ and separable-bilinear of size $\sigma$, respectively. The SIFT descriptor is a 128-dimensional vector that is a concatenation of a normalized $4 \times 4$ cells with 8 bins. DSP-SIFT is a modified form of the standard SIFT, obtained by pooling gradient orientations from different scaled domain sizes, instead of spatial space. The formula is given by:

$$
h_{D S P}(\theta, I)(x)=\int h_{S}(\theta, I, \sigma)(x) \epsilon_{s}(\sigma) d \sigma,
$$

where $E$ is an exponential unilateral density function and $s$ is the size-pooling scale. DSP-SIFT kept the same descriptor dimension but more suitable for searching correspondence in the presence of occlusions.

After extracting feature points and their DSP-SIFT descriptors between subsequent images, a perspective homography constrain was applied to filter outliers by mapping one image to another. 'Inliers' are considered if the co-planar feature points between subsequent images are fully matched or within a small displacement. The Random sample consensus (RANSAC) algorithm was applied to optimize the homography transformation under the over-constrained degree of freedom problem. RANSAC uses iterative calculations to estimate optimal parameters of homography matrix ( $8 \mathrm{DOF}$ ) from observations, and removed outliers from the matching process.

3.2.2 Visual-inertial trajectory estimation We implemented a multi-state constraint Kalman filter to combine the IMU and monocular vision-based measurements. Tracked pose of the CameraIMU sensor are presented based on the Earth-Centered, EarthFixed (ECEF) coordinates. Initial camera poses were given by the EKF propagating state and covariance, updated from multiobserved feature points, assuming that $\mathrm{N}$ camera poses are included in the EKF state at each time step $k$. The state and errorstate vector are:

$$
\begin{gathered}
\hat{X}_{k}=\left[\hat{X}_{I M U, k}^{T} \hat{q}_{C_{1} G}^{T} \hat{p}_{C_{1} G}^{T} \hat{q}_{C_{N} G}^{T} \hat{p}_{C_{N} G}^{T}\right]^{T}, \\
\tilde{X}_{k}=\left[\tilde{X}_{I M U, k}^{T} \delta \Theta_{C_{1}}^{T} \tilde{p}_{C_{1} G}^{T} \cdots \delta \Theta_{C_{N}}^{T} \tilde{p}_{C_{N} G}^{T}\right]^{T}, \\
X_{I M U, k}=\left[q_{I G, k}^{T} b_{g, k}^{T} b_{a, k}^{T} p_{I G, k}^{T}\right]^{T}, \\
\tilde{X}_{I M U}=\left[\delta \Theta_{I, k}^{T} \tilde{b}_{g, k}^{T} \tilde{b}_{a, k}^{T} \tilde{p}_{I G, k}^{T}\right]^{T},
\end{gathered}
$$

where $q_{I G, k}$ is the unit quaternion representing the rotation from global frame $G$ to the IMU frame $I$ at time $k . p_{I G, k}$ is the IMU position with respect to global frame. $b_{g}$ and $b_{a}$ are the biases of gyroscope and accelerometer measurements, respectively. $\delta \Theta$ described the attitude errors is a minimal representation. To sum up, at time $k$, the full state of the KF consists of the 13dimensional IMU state and $N \times 7$-dimensional past camera poses in which active feature tracks were visible. In the propagation step, the filter propagation equations are derived by discretization of the continuous-time IMU system model, the linearized continuous-time model is formulated:

$$
\begin{gathered}
\dot{X}_{I M U}=F \tilde{X}_{I M U}+G n_{I M U}, \\
F=\left[\begin{array}{cccc}
-\hat{\omega}^{x} & -I_{3} & 0_{3 \times 3} & 0_{3 \times 3} \\
0_{3 \times 3} & 0_{3 \times 3} & 0_{3 \times 3} & 0_{3 \times 3} \\
0_{3 \times 3} & 0_{3 \times 3} & 0_{3 \times 3} & 0_{3 \times 3} \\
-\hat{C}_{I G}^{T} \hat{v}^{x} & 0_{3 \times 3} & -\hat{C}_{I G}^{T} & 0_{3 \times 3}
\end{array}\right], \\
G=\left[\begin{array}{cccc}
-I_{3} & 0_{3 \times 3} & 0_{3 \times 3} & 0_{3 \times 3} \\
0_{3 \times 3} & I_{3} & 0_{3 \times 3} & 0_{3 \times 3} \\
0_{3 \times 3} & 0_{3 \times 3} & 0_{3 \times 3} & I_{3} \\
0_{3 \times 3} & 0_{3 \times 3} & -\hat{C}_{I G}^{T} & 0_{3 \times 3}
\end{array}\right],
\end{gathered}
$$

where $n_{I M U}$ is the system noise. The covariance matrix of $n_{I M U}$ is computed off-line during sensor calibration. The matrices $F$ and $G$ are the Jacobians; $I_{3}$ is $3 \times 3$ identity matrix; $\omega$ and $v$ are $3 \times 3$ rotational velocity and linear velocity matrix, respectively; $C_{I G}^{T}$ is the rotation matrix corresponding to $q_{I G}^{T}$.

Every IMU readings are used for the state propagation in the EKF. Moreover, the EKF covariance matrix has to be propagated every $\mathrm{k}+1$ steps. State covariance is a $(12+6 N) \times(12+6 N)$ matrix and state covariance propagation is given by:

$$
\begin{gathered}
P_{k \mid k}=\left[\begin{array}{ll}
P_{I I_{k \mid k}} & P_{I C_{k \mid k}} \\
P_{I C_{k \mid k}}^{T} & P_{C C_{k \mid k}}
\end{array}\right], \\
P_{k+1 \mid k}=\left[\begin{array}{cc}
P_{I I_{k \mid k}} & \Phi\left(t_{k}+T, t_{k}\right) P_{I C_{k \mid k}} \\
P_{I C_{k \mid k}}^{T} \Phi\left(t_{k}+T, t_{k}\right)^{T} & P_{C C_{k \mid k}}
\end{array}\right],
\end{gathered}
$$

where $\Phi\left(t_{k}+T, t_{k}\right)$ is the state transition matrix. $P_{I I}$ is the $12 \times 12$ covariance matrix of the current IMU state; $P_{C C}$ is the $6 N \times 6 N$ covariance matrix of the camera poses; and $P_{I C}$ is the $12 \times 6 N$ correlation between the errors in the IMU state and the camera pose estimates.

When capturing a new video frame, the camera pose estimation is computed from the IMU pose estimation. This camera pose estimate is appended to the state vector, and the covariance matrix of the EKF is augmented as:

$$
\hat{P}_{k} \leftarrow\left[\begin{array}{c}
I_{12+6 N} \\
J_{k}
\end{array}\right] \hat{P}_{k}\left[\begin{array}{c}
I_{12+6} \\
J_{k}
\end{array}\right]^{T},
$$




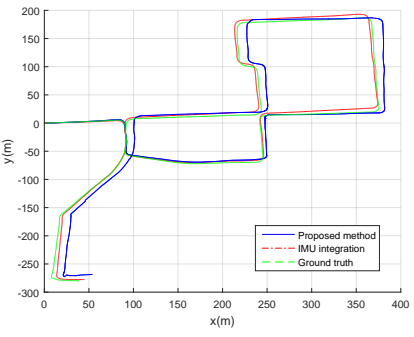

(a) 2D trajectory

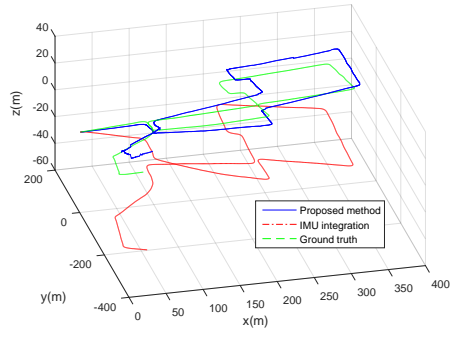

(b) 3D trajectory

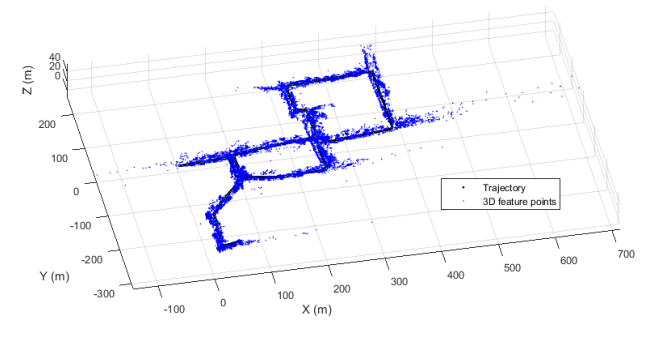

(c) $3 \mathrm{D}$ point cloud

Figure 7: The trajectory estimated by our method from 3D point cloud

$$
J_{k}=\left[\begin{array}{cccc}
\hat{C}_{C I, k} & 0_{3 \times 6} & 0_{3 \times 3} & 0_{3 \times 6 N} \\
\left(\hat{C}_{I G, k}^{T} P_{I, k}^{C I}\right)^{\times} & 0_{3 \times 6} & I_{3} & 0_{3 \times 6 N}
\end{array}\right],
$$

where $J_{k}$ is the Jacobian matrix, $f_{j}$ is a feature point that has been observed from a set of $M_{j}$ camera poses. We use an inverse depth least-squares Gauss-Newton optimization method to estimate three-dimensional location of $f_{j}$. The residual for each measurement is independent of the errors in the feature coordinates, and thus EKF updates can be performed based on it. $H_{X_{i}}$ and $H_{f_{i}}$ are the Jacobians of the measurement $z_{i}$ with respect to the state and the feature position, respectively. Kalman gain can then be calculated as $\left.K=P T_{H}^{T}\left(T_{H} P T_{H}^{T}+R_{n}\right)^{(}-1\right)$, where $T_{H}$ is an upper triangular matrix from QR decomposition of the matrix $H_{X}$. Finally, the state covariance matrix is updated according to

$$
P_{k+1 \mid k}=\left(I_{\xi}-K T_{H}\right) P_{k+1 \mid k}\left(I_{\xi}-K T_{H}\right)^{T}+K R_{n} K^{T} .
$$

\section{EXPERIMENTS}

In our experiments we evaluate our visual odometry pipeline in 4.1 and demonstrate our geo-localization approach in 4.2 using maps acquired from OpenStreetMap and GIS from different urban and semi-urban regions in different parts of the world.

\subsection{Visual Odometry}

The IMU data provided in this dataset was recorded by OXTS $R T$ 3003, containing acceleration and angular rate around three axes with $100 \mathrm{~Hz}$ sampling rate. Video frames were captured using Point Grey Flea 2 (FL2-14S3M-C) in gray-scale with 1242 $\times 375$ pixels resolution (after calibration). Shutter time adjusted dynamically (maximum shutter time: $2 \mathrm{~ms}$ ) and triggered at 10 $\mathrm{Hz}$ frequencies. This dataset provides well-calibrated camera intrinsic parameters and camera-IMU rotation and translation matrix. The synchronized IMU and video frames are at $10 \mathrm{~Hz}$ for this study.

Figure 7 demonstrated the trajectory estimated for a long term tracking case. The translational average root mean squared error (ARMSE) is $4.154 m$, rotational ARMSE is $0.026 m$, and final translational error is $12.149 \mathrm{~m}$. The accuracy for the estimated is capable for proposed GIS path searching algorithm. Figure 8 demonstrated the comparison between proposed method, IMU integration and MSCKF implemented by (Clement et al., 2015) on case no. 0051. Table 1 quantified the error ARMSE with two other visual-initial approaches, MSCKF and SWF.

\subsection{Geo-localization}

We found that abstraction of trajectory and map to graph structure should we based on the scale and frequency of occurrence of significant features. To improve efficiency of the implementation

\begin{tabular}{|c|l|l|l|l|l|l|}
\hline \multicolumn{2}{|c|}{ Dataset ID } & 0001 & 0005 & 0035 & 0051 & 0095 \\
\hline IMU & Tra. ARMSE & 0.784 & 0.965 & 0.263 & 2.255 & 1.945 \\
\hline & Rot. ARMSE & 0.003 & 0.015 & 0.009 & 0.008 & 0.007 \\
\hline & Final Tra. Err. & 2.532 & 3.109 & 0.469 & 6.335 & 7.592 \\
\hline MSCKF & Tra. ARMSE & 0.449 & 1.217 & 0.268 & 2.310 & 1.324 \\
\hline & Rot. ARMSE & 0.007 & 0.023 & 0.009 & 0.028 & 0.046 \\
\hline & Final Tra. Err. & 1.130 & 2.453 & 1.879 & 9.294 & 3.628 \\
\hline SWF & Tra. ARMSE & 0.432 & 0.968 & 0.279 & 1.178 & 1.900 \\
\hline & Rot. ARMSE & 0.007 & 0.023 & 0.006 & 0.016 & 0.010 \\
\hline & Final Tra. Err. & 1.136 & 3.712 & 0.719 & 2.167 & 7.579 \\
\hline Our & Tra. ARMSE & 0.342 & 1.115 & 0.269 & 2.875 & 1.885 \\
\hline & Rot. ARMSE & 0.009 & 0.011 & 0.011 & 0.032 & 0.146 \\
\hline & Final Tra. Err. & 1.797 & 3.053 & 1.577 & 4.742 & 5.738 \\
\hline
\end{tabular}

Table 1: Comparison of translation ARMSE, rotation ARMSE, and final translation error of proposed method, MSCKF, and SWF on KITTI dataset. (units: $m$ )

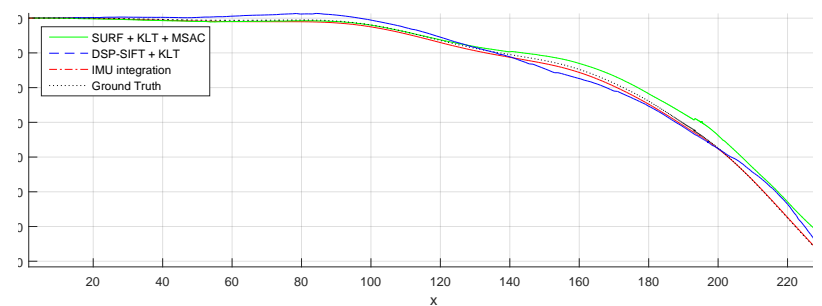

Figure 8: The comparison between proposed method, IMU integration and MSCKF on case no.0051.

we begin with an analysis of the topology of map data acquired from OpenStreetMap, described in 4.2.1. There results were used to tune the parameters of our geo-localization pipeline which is evaluated on several instances of regions from different parts of the world in 4.2.2.

4.2.1 Map Features The number and location of nodes and edges of $\mathcal{G}_{\mathcal{M}}$ abstracted from the $O S M$ map should be based on the degree of quantization that reduces the computational complexity while preserving the uniqueness of different edges and subsequently path graphs $\mathcal{G}_{\mathcal{P}}$ so that the search and converge to the best matching $\mathcal{G}_{\mathcal{P}}^{*}$ as quickly as possible. In Figure 9, we show an example of two cities, Washington DC and Karlsruhe, Germany, which have different types of transport network topologies. Washington DC, in Figure 9c, shows a regular lattice grid structure, where edge lengths $\ell$ and orientations $\theta$ have similar across several nodes in the graph. In comparison, Karlsruhe, Germany has a semi-urban type of transport network seen in Figure 9a with a spaghetti structure. The graphs in Figures $9 \mathrm{~b}$ and $9 \mathrm{~d}$ show the $(\ell, \theta)$-histogram for Karlsruhe and Washington DC respectively, where the different between the transport network topologies is evident. 

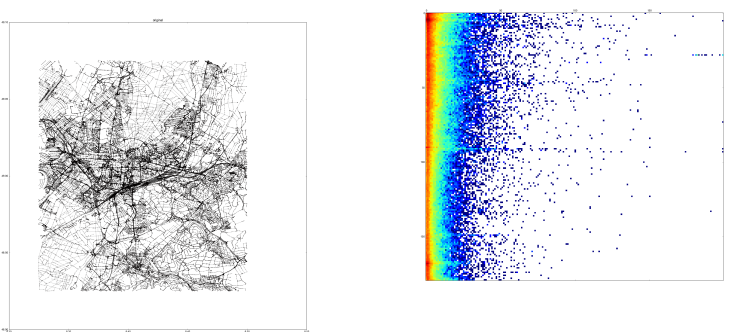

(b) $(\ell, \theta)$-histogram for map of (a) $\mathcal{G}_{\mathcal{M}}$ of Karlsruhe, Germany Karlsruhe, Germany
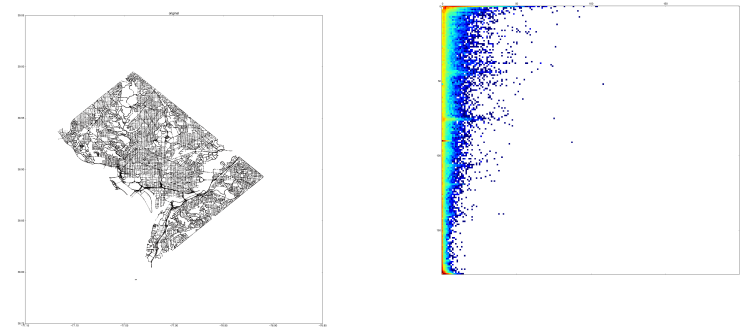

(c) $\mathcal{G}_{\mathcal{M}}$ of Washington DC

(d) $(\ell, \theta)$-histogram for Washington DC

Figure 9: Analysis of $\mathcal{G}_{\mathcal{M}}$ of urban and semi-urban cities of Washington DC and Karlsruhe, Germany. The graphs shows the $(\ell, \theta)$-histogram of the length $\ell$ and orientations $\theta$ of the edges of $\mathcal{G}_{\mathcal{M}}$ (columns correspond to edge lengths $\ell$ and rows correspond to edge orientations $\theta$ ). The lattice structured urban and spaghetti structured semi-urban graphs show different histograms reflecting the inherent difference in these two types of maps.

4.2.2 Trajectory search We acquired maps for regions of interest from OpenStreetMap and GIS database to evaluate the performance of our approach for different types of transport network topologies including urban, semi-urban and country roads, and scale of the search region. We selected maps for: 'Franklin county, Ohio' which provides a typical U.S. county sized map; 'Washington DC', which is a highly urban region with a lattice grid structured road network; and 'Montpellier, France', which is a semi-urban and country spaghetti shaped road network of a state sized region. Some of the results of our experiments for each of these regions is illustrated in Figure 10. Initially, the estimated trajectory $\mathcal{G}_{\tau}^{t_{0}}$ is short as the mobile platform begins moving. We compute $\mathcal{G}_{\mathcal{P}}$ and associated similarity score $\mathcal{S}\left(\mathcal{G}_{\tau}^{t_{0}}, \mathcal{G}_{\mathcal{P}}\right)$ and there are several matching paths, shown in the first column of the figure for each region. As the estimated trajectory grows $\mathcal{G}_{\tau}^{t_{n}}$ the number of candidate paths reduce based on our empirically determined threshold, shown in the center column in the figure. Subsequently, a single path with the highest similarity score remains, which is our matched path in the map graph. The right column for each region in the figure shows the matched path in the graph for the corresponding mobile platform trajectory. In our experiments we found that quick search depends on the uniqueness of the trajectory and the nature of the map graph.

\section{CONCLUSION}

We have presented a novel approach for geo-localizing position of a mobile platform. We have proposed a modified approach to classical visual odometry pipeline with map data from public domain sources like OpenStreetMaps and GIS databases in a unified framework. We have described the algorithmic and implementation details of our method and demonstrated it on several different types of maps from different regions of the world. We have demonstrated excellent results on our proposed visual odometry pipeline on benchmark KITTI dataset. Our results show that the proposed system is able to provide fully automatic global localization at a low infrastructural cost. Building on this work, we plan to further investigate the use of maps, especially different layers of a GIS database such as building, hydrology, relief maps, etc. We also plan to integrate outdoor and indoor GPS-denied navigation for seamless uninterrupted geo-navigation in all locations and environment conditions.

\section{REFERENCES}

Civera, J., Grasa, O. G., Davison, A. J. and Montiel, J. M. M., 2010. 1 point ransac for extended kalman filtering: Application to realtime structure from motion and visual odometry. Journal of Field Robotics 27(5), pp. 609-631.

Clement, L. E., Peretroukhin, V., Lambert, J. and Kelly, J., 2015. The battle for filter supremacy: A comparative study of the multistate constraint kalman filter and the sliding window filter. In: Computer and Robot Vision (CRV), 2015 12th Conference on.

Dong, J. and Soatto, S., 2015. Domain-size pooling in local descriptors: Dsp-sift. In: Computer Vision and Pattern Recognition (CVPR), 2015 IEEE Conference on, pp. 5097-5106.

Engel, J., Stückler, J. and Cremers, D., 2015. Large-scale direct slam with stereo cameras. In: IEEE/RSJ International Conference on Intelligent Robots and Systems.

Hentschel, M. and Wagner, B., 2010. Autonomous robot navigation based on OpenStreetMap geodata. Intelligent Transportation Systems (ITSC), 2010 13th International IEEE Conference on pp. 1645-1650.

Lategahn, H., Geiger, A. and Kitt, B., 2011. Visual SLAM for autonomous ground vehicles. Proceedings - IEEE International Conference on Robotics and Automation pp. 1732-1737.

Mourikis, A. and Roumeliotis, S., 2007. A multi-state constraint kalman filter for vision-aided inertial navigation. In: Robotics and Automation (ICRA), 2007 IEEE International Conference on, IEEE, pp. 3565-3572.

Ovrén, H. and Forssén, P.-E., 2015. Gyroscope-based video stabilisation with auto-calibration. In: Robotics and Automation (ICRA), 2015 IEEE International Conference on, IEEE, pp. 2090-2097.

Paul, R. and Newman, P., 2010. FAB-MAP 3D: Topological mapping with spatial and visual appearance. Proceedings - IEEE International Conference on Robotics and Automation pp. 26492656.

Senlet, T. and Elgammal, A., 2011. A framework for global vehicle localization using stereo images and satellite and road maps. In: 2011 IEEE International Conference on Computer Vision Workshops (ICCV Workshops), pp. 2034-2041.

Strelow, D. and Singh, S., 2004. Motion estimation from image and inertial measurements. The International Journal of Robotics Research 23(12), pp. 1157-1195.

Vaca-Castano, G., Zamir, A. R. and Shah, M., 2012. City scale geo-spatial trajectory estimation of a moving camera. Proceedings of the IEEE Computer Society Conference on Computer Vision and Pattern Recognition pp. 1186-1193. 

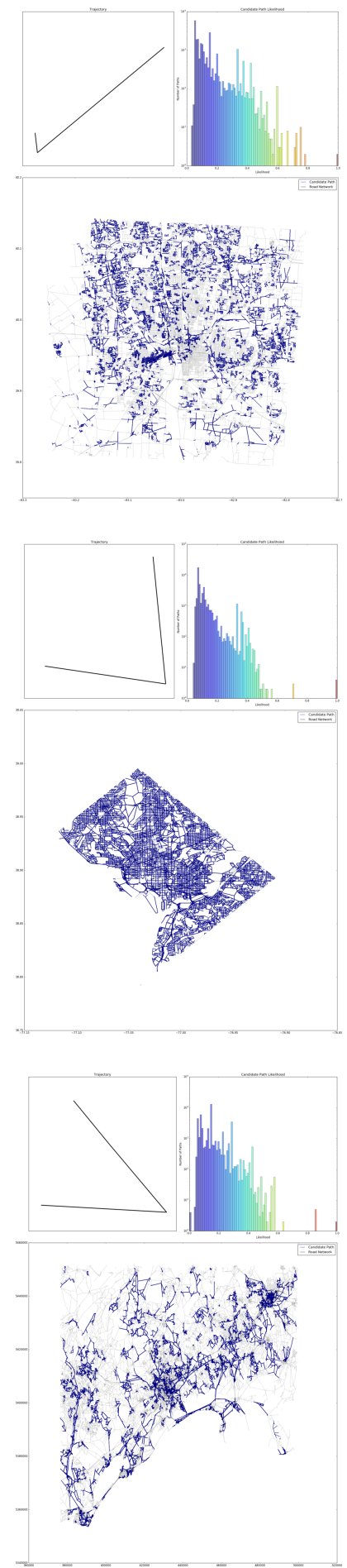
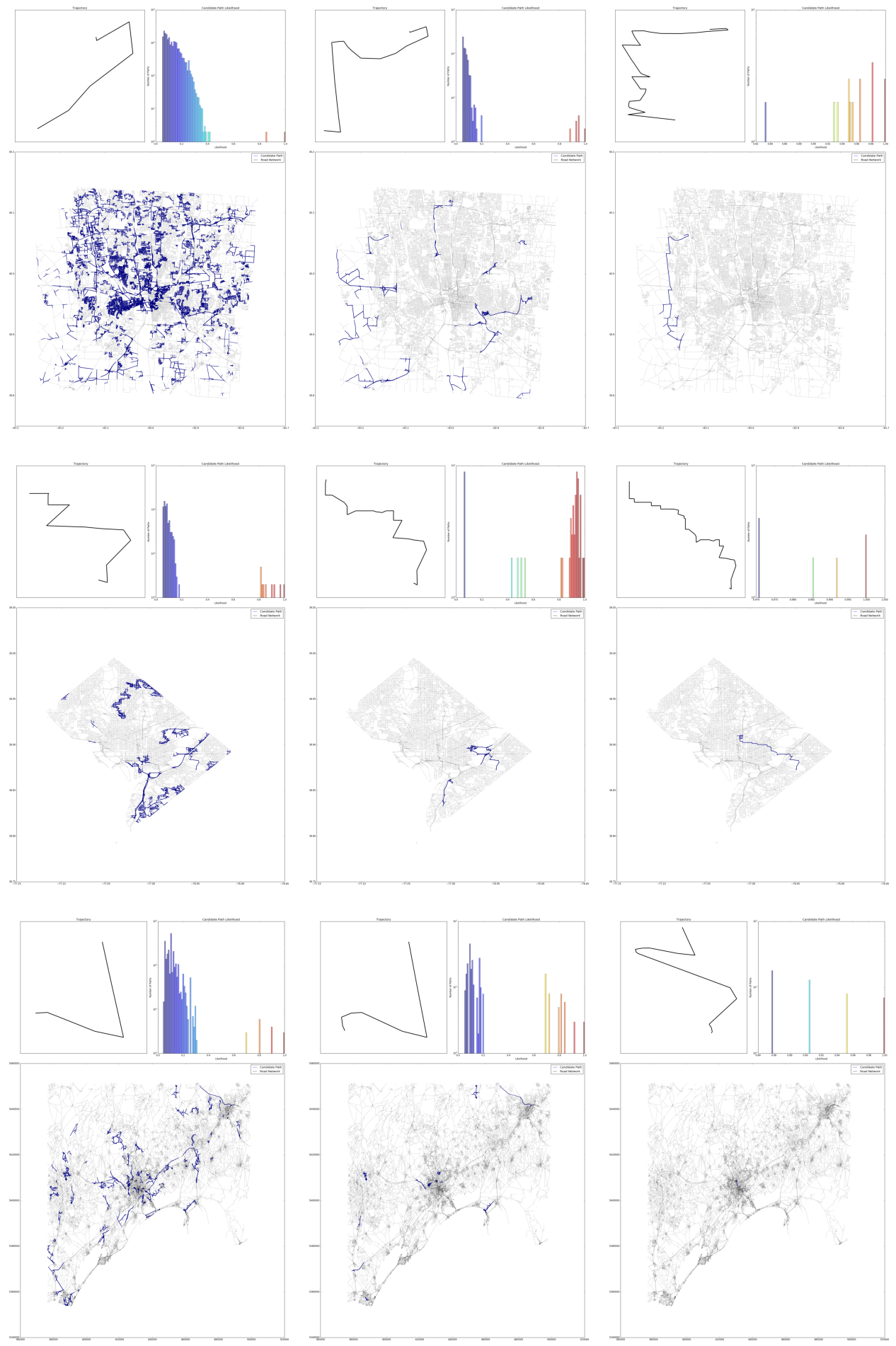

Figure 10: Geo-localization of mobile platform trajectory, from top to bottom for the geographic regions of 'Franklin County, Ohio', 'Washington DC, US', and 'Montpellier, France'. The estimated trajectory of the mobile platform is shown on the top left; the histogram on the top right shows the match score of each candidate path; and the map shows the current candidate paths. In the first column, the initial trajectory $T_{V O}$ is small and several candidate paths $\mathcal{G}_{\mathcal{P}}$ are spawned. The similarity match score is shown in the histogram graph. As $T_{V O}$ grows several $\mathcal{G}_{\mathcal{P}}$ are pruned since $\mathcal{S}\left(\mathcal{G}_{\tau}, \mathcal{G}_{\mathcal{P}}\right) \leq$ threshold. Finally, the best matching path $\mathcal{G}_{\mathcal{P}}^{*}$ remains, shown in the right column, which is used for inferring geo-location of the mobile platform. 